\title{
The Hydrogen Clouds in the Galactic Halo
}

\author{
Felix J. Lockman \\ National Radio Astronomy Observatory, Green Bank WV, USA
}

\begin{abstract}
New $21 \mathrm{~cm}$ observations with the Green Bank Telescope show that a significant fraction of the HI in the inner Galaxy's halo $\sim 1 \mathrm{kpc}$ from the midplane exists in the form of discrete clouds. Some look very much like a Spitzer (1968) "standard" diffuse cloud but with their HI in two phases. They mark the transition between the neutral disk and the highly ionized halo. The dominant motion of the clouds is Galactic rotation, but some have random velocities of as much as $50 \mathrm{~km} \mathrm{~s}^{-1}$. They are part of the Galaxy and are not related to high-velocity clouds, yet their origin is obscure.
\end{abstract}

\section{Introduction}

In the inner parts of the Milky Way most of the hydrogen is confined to a narrow disk which establishes a plane of fundamental physical significance (Blaauw et al. 1960). But the more we look out of the disk, the more HI we see at curious locations. Many mechanisms can lift HI from below, and the Milky Way, like other galaxies, is probably accreting gas from above, so the halo is likely an untidy place, containing HI that 1) has been pushed outward by mechanical and photon energy from young stars and supernovae, 2) has condensed from a halo of very hot gas and now returns in a cool phase to the plane, 3) is falling into the Milky Way for the first time, having been stripped from neighboring galaxies like the Magellanic Stream, or coming in as high-velocity clouds (Shapiro \& Field 1976; Heiles 1984; Norman \& Ikeuchi 1989; Houck \& Bregman 1990; Wakker et al. 1999; Sancisi et al. 2001; de Avillez \& Berry 2001; Konz et al. 2002; Putman et al. 2003).

This paper discusses new observations of the HI at some distance from the Galactic plane which show the organization of halo HI for the first time, but at present raise more questions than answers.

\section{The Structure of HI Above the Disk}

Previous studies of $21 \mathrm{~cm}$ emission in the inner Galaxy were able to detect neutral gas to considerable distances from the Galactic plane (Oort 1961; Lockman 1984), but lacked the angular resolution to determine its structure. That situation has now changed because of the Green Bank Telescope (GBT), a 100-meter diameter offset paraboloid antenna, which has very good sensitivity, high dynamic range, and an angular resolution of about $9^{\prime}$ in the $21 \mathrm{~cm}$ line. 


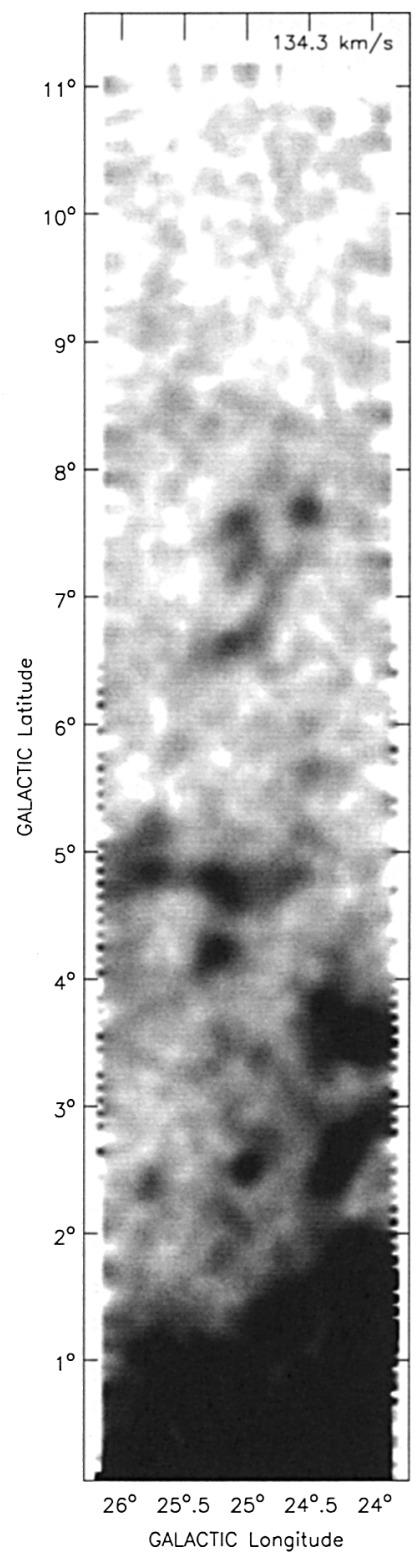

Figure 1. HI emission above the Galactic plane in a region around $\ell=25^{\circ}$ as observed with the GBT at $9^{\prime}$ angular resolution. The data cover $V_{L S R}=134 \pm 1 \mathrm{~km} \mathrm{~s}^{-1}$, which is at the tangent point in this direction, where one degree in either coordinate corresponds to a linear distance of $135 \mathrm{pc}$. The clouds at $b \approx+7.6$ are $1 \mathrm{kpc}$ above the plane. 
Figure 1 shows recent GBT observations of HI above the Galactic plane around $\ell=25^{\circ}$. There is some HI which might be a diffuse component falling off gradually with latitude, but the most striking features are the clouds which stand out above the background. These clouds are well-defined in position and velocity, are several orders of magnitude denser than their surroundings, and may contain a significant fraction of the neutral gas in the halo. Similar clouds are seen throughout the inner Galaxy (Lockman 2002). The halo clouds follow Galactic rotation, so their origin is tightly tied to events in the disk and lower halo. In the rest of this paper I will discuss a few features of this population and give provisional answers to some of the most obvious questions.

\section{A Halo Cloud and its Surroundings}

Figure 2 shows a series of GBT HI spectra across the halo cloud at $25.1+7.6$ from Fig. 1. The spectra show: 1) this cloud is not sitting on a plateau of $\mathrm{HI}$ emission at the same velocity - it contains most of the HI emission at its velocity in its vicinity. 2) At the edge of the cloud the line shape changes. Closer inspection shows that this cloud has two line components, one with a FWHM of $8 \mathrm{~km} \mathrm{~s}^{-1}$, and the other, at an identical velocity, with a FWHM of $26 \mathrm{~km} \mathrm{~s}^{-1}$. The narrower line is confined to the center of the cloud. This cloud (and many others) thus contains HI in at least two phases, a condition which is observed in other locations (Liszt 1983), and which can occur only over a restricted range of physical conditions (Field, Goldsmith \& Habing 1969; Wolfire et al. 1995). 3) This cloud appears to be connected to several others by a narrow filament which has a linewidth similar to the broad component of the cloud. Such connections are also seen between a few other clouds, but not all. The cloud has a mass in $\mathrm{HI} \approx 400 M_{\circ}$, a size of $50 \times 35 \mathrm{pc}$, and a peak $N_{H} \geq 3 \times 10^{19} \mathrm{~cm}^{-2}$.

\section{The Velocity of the Clouds}

A set of about 40 halo clouds near longitude $29^{\circ}$, all more than $500 \mathrm{pc}$ from the plane, were studied in some detail (Lockman 2002). Here I will concentrate on their velocities. Fig. 3 shows the number of clouds in intervals of $V_{L S R}$. The distribution implies a cloud-to-cloud velocity dispersion of several tens of $\mathrm{km} \mathrm{s}^{-1}$. Verification of this will require more careful analysis, for at the lower velocities clouds are so common that they are confused and probably undercounted. Nonetheless, some of the "fastest" clouds have random velocities of $>40 \mathrm{~km} \mathrm{~s}^{-1}$. The fastest clouds do not have a preferential location with respect to the plane, but seem to be found at all latitudes. The only property of the clouds which is correlated with velocity is mass: the higher the random velocity the smaller the mass. Thus, in the halo the HI mass is concentrated toward velocities permitted by Galactic rotation, just as it is in the disk.

It was suggested some time ago that the Galaxy contained a population of "fast" interstellar clouds whose random velocities might carry them into the halo (Radhakrishnan \& Srinivasan 1980; Kulkarni \& Fich 1985; Lockman \& Gehman 1991), but individual clouds could not be resolved in the older data. Now we know of distinct HI clouds with random velocities of several tens of 


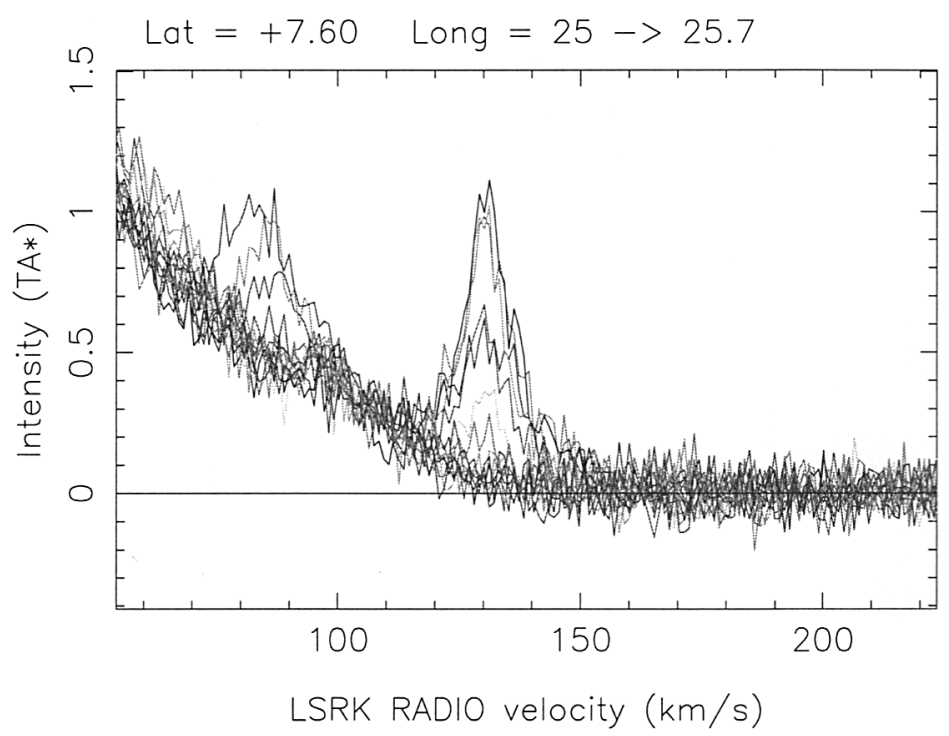

Figure 2. GBT $21 \mathrm{~cm}$ spectra across the halo cloud at latitude 7.6 between $25^{\circ} \leq \ell \leq 25^{\circ} .7$ at $3^{\prime}$ spacing. There is little emission away from the cloud. The line changes shape at the cloud edge.

$\mathrm{km} \mathrm{s}^{-1}$ not only in the halo, but at low latitude as well (Lockman \& Stil 2004). This population may be quite widespread in the inner Galaxy, and may also be linked to the broad HI profile components found by Kalberla et al. (1998).

\section{Comments on the Origin of the Halo Clouds}

Because the halo clouds are firmly tied to Galactic rotation even though some have a large random velocity, they cannot be extragalactic material falling into the disk, like some high-velocity clouds (e.g., Wakker et al. 1999). So where did the halo clouds come from? Possible sources were discussed in $\S 1$ : all involve massive stars and supernovae which can push HI outward and also create hot gas for a Galactic "fountain". There is ample evidence that these processes are at work (e.g., Savage 1995; Sembach et al. 2003), but it is not obvious to me that the basic theoretical models have been developed far enough to be tested against the new data.

The clouds detected so far look approximately spheroidal, even when observed at $1^{\prime}$ angular resolution (Liszt, Lockman \& Rupen, in preparation). They look stable, though their dynamical time (size/linewidth) is only a few $10^{6}$ years. Does something surround the clouds keeping them in equilibrium? At a distance of $1 \mathrm{kpc}$ from the Galactic plane the average HI density in the inner Galaxy is expected to be $0.005 \mathrm{~cm}^{-3}$ (Dickey \& Lockman 1990), while the average density of $\mathrm{H}^{+}$will be about twice this value, though it probably fills only a fraction of the volume (Reynolds 1997). A typical halo cloud has $\langle n\rangle \approx 0.25 \mathrm{~cm}^{-3}$ (Lockman 2002) suggesting that if most of the halo HI is in clouds, the filling factor of 


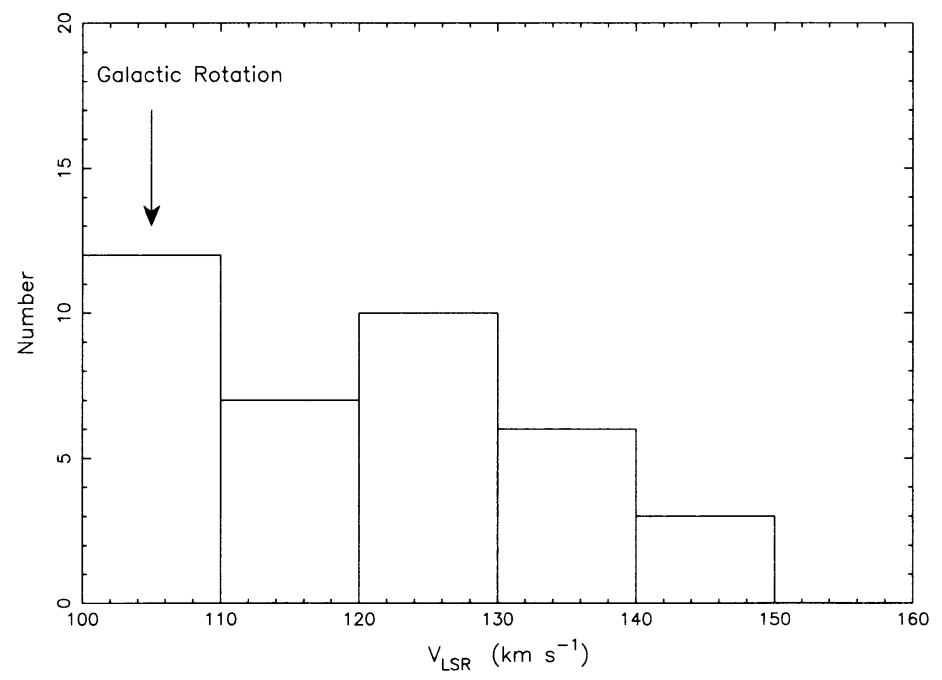

Figure 3. The distribution of $V_{L S R}$ for halo HI clouds in a sample near $\ell=29^{\circ}$ (Lockman 2002). These clouds are at a median distance of $-940 \mathrm{pc}$ from the plane. The arrow marks the maximum velocity expected for Galactic rotation in this direction, as determined from the velocity of molecular clouds in the disk (Clemens 1985).

neutral gas is only a few percent. The remainder of the space may be filled with very hot gas which provides confinement of the halo clouds if, indeed, they are pressure-confined, but this an assumption not an observation (e.g., Cox 2000). One certainty is that the clouds have too little HI mass to be self-gravitating.

Whatever holds them together, at $1 \mathrm{kpc}$ height the halo clouds are denser than their surroundings by more than an order of magnitude and left to themselves will plunge toward the plane. It is plausible that they have a vertical velocity of at least the same magnitude as their random line-of-sight velocity, i.e., several tens of $\mathrm{km} \mathrm{s}^{-1}$, though whether the dominant motion is toward the midplane as would be expected from fountain models, or is in equilibrium (some clouds rising, others falling), cannot be resolved by the data in hand.

\section{Prospects}

The study of halo clouds is just beginning. They are likely to be sensitive tracers of physical conditions in their vicinity and could be used as probes of their environment. Moreover, they are the best examples known of diffuse interstellar clouds of the kind envisioned by Spitzer (1968), though they lie in the halo, not the disk. In fact, the halo clouds are the only diffuse interstellar clouds which show well-defined boundaries in position and velocity when mapped in HI (cf., Kulkarni \& Heiles 1988). They may be useful not only for study of conditions in the halo, but for study of fundamental interstellar processes. The GBT data 
make it clear that the neutral halo has more structure, and is more amenable to analysis, than had been suspected before.

Acknowledgments. The National Radio Astronomy Observatory is operated by Associated Universities, Inc., under a cooperative agreement with the National Science Foundation.

\section{References}

Blaauw,A., Gum,C.S., Pawsey,J.L., \& Westerhout,G. 1960, MNRAS, 121, 123

Clemens, D.P. 1985, ApJ, 295, 422

Cox, D.P. 2000, Rev. Mex. A. A., 9, 14

de Avillez, M.A., \& Berry, D.L. 2001, MNRAS, 328, 708

Dickey, J.M., \& Lockman, F.J. 1990, ARAA, 28, 215

Field, G.B., Goldsmith, D.W., \& Habing, H.J. 1969, ApJ, 155, L149

Heiles, C. 1984, ApJS, 55, 585

Houck, J.C., \& Bregman, J.N. 1990, ApJ, 506, 521

Kalberla, P.M.W. et al. 1998, A\&A, 332, L61

Konz, C., Brüns, C., \& Birk, G.T. 2002, A\&A, 391, 713

Kulkarni, S.R., \& Fich, M. 1985, ApJ, 289, 792

Kulkarni, S. \& Heiles, C.E. 1988, in Galactic and Extragalactic Radio Astronomy, ed. G.L. Verschuur \& K.I. Kellermann, Springer, p. 95

Liszt, H.S. 1983, ApJ, 275, 163

Lockman, F.J. 1984, ApJ, 283, 90

Lockman, F.J. 2002, ApJ, 580, L47

Lockman, F.J., \& Gehman, C.S. 1991, ApJ, 382, 182

Lockman, F. J. \& Stil, J. R. 2004, in press (astro-ph 0310762)

Norman, C. \& Ikeuchi, S. 1989, ApJ, 345, 372

Oort, J. 1961, in The Distribution and Motion of Interstellar Matter in Galaxies, ed. L. Woltjer, (Benjamin:New York), p. 71

Putman, M.E., et al. 2003, ApJ, 586, 170

Radhakrishnan, V., \& Srinivasan, G. 1980, J. Astr. Ap., 1, 47

Reynolds, R.J. 1997, in Physics of Galactic Halos, ed. H. Lesch, R-J. Dettmar, U. Mebold \& R. Schlickeiser, Akademie Verlag: Berlin, p. 57

Sancisi, R., Fraternali, F., Oosterloo, T., \& van Moorsel, G. 2001, in Galaxy Disks and Disk Galaxies, ASP Conf. Ser. 230, p. 111

Savage, B.D. 1995, in The Physics of the Interstellar Medium and Intergalactic Medium, ASP Conf. Ser. 80, p. 233

Sembach, K.R. et al. 2003, ApJS, 146, 165

Shapiro, P.R., \& Field, G.B. 1976, ApJ, 205, 762

Spitzer, L. 1968, Diffuse Matter in Space, (Wiley:Interscience)

Wakker, B.P. et al. 1999, Nature, 402, 388

Wolfire, M.G., et al. 1995, ApJ, 453, 673 